\title{
THz Driven Accelerators
}

\author{
Franz X. Kärtner \\ Center for Free-Electron Laser Science and Deutsches Elektronen-Synchrotron (DESY), Notkestraße 85, 22607 Hamburg, Germany \\ Department of Physics and Center for Ultrafast Imaging, University of Hamburg, Luruper Chaussee 149, 22761 Hamburg, Germany \\ e-mail address: franz.kaertner@desy.de
}

\begin{abstract}
High-energy THz pulse generation and approaches towards linear THz accelerators are discussed and demonstrated. First results from $\mathrm{THz}$ guns and accelerators are shown and concepts for compact X-ray sources based on these devices are presented.

OCIS codes: (320.0320) Ultrafast Optics; (190.4410) Nonlinear optics, parametric processes; (190.7110) Ultrafast nonlinear optics.
\end{abstract}

\section{Introduction}

Today, high brightness and highly relativistic electron beams are generated by circular or linear accelerators (LINAC) typically operating with 1-3 GHz accelerating frequencies, and, approaches towards X-band frequencies in the $10 \mathrm{GHz}$ range are maturing. The achievable accelerating gradients are limited by field emission from cavity walls influenced by pulsed heating to several tens of $\mathrm{MV} / \mathrm{m}$ in the case of low frequencies and up to $100 \mathrm{MV} / \mathrm{m}$ in the case of X-band frequencies. Short electron bunches are typically created by photoemission from the cathode in the presence of a strongly accelerating field followed by bunch compression. Low charge bunches, 1-10 pC, may be compressed to durations down to $3-10 \mu \mathrm{m}$ (or $10-30 \mathrm{fs}$ in pulse duration). At a given accelerating field strength and RF frequency, compression is limited by space charge. With higher operating frequency, higher gradients in the accelerating electric field arise which enhances velocity bunching and leads to the generation of shorter electron bunches. These short electron bunches can be used for ultrafast electron diffraction or intense X-ray production. Choosing an operating frequency of the accelerator in the $\mathrm{THz}$ range, i.e. here $0.1-0.5 \mathrm{THz}$ and using multi-cycle to single-cycle pulses, accelerating fields in the 0.3 to $1 \mathrm{GV} / \mathrm{m}$ range are sustainable and bunch compression to the sub-femtosecond level of significant charge on the order of $\sim 1 \mathrm{pC}$ becomes possible. To drive electron guns and accelerators high-energy single-cycle and multi-cylce THz pulses in the $\mathrm{mJ}$ and tens of $\mathrm{mJ}$ range, respectively, are necessary. Here, we discuss approaches towards this goal and first results achieved sofar.

\section{High energy THz generation}

The traditional approach towards generation of single-cycle $\mathrm{THz}$ pulses is via optical rectification in Lithium Niobate using the tilted pulse front technique (TPFT) [1]. Using 1 micron, sub-ps pulses up to $0.4 \mathrm{~mJ} \mathrm{THz}$ pulses have been generated with up to $0.8 \%$ efficiency albeit the center frequency moved below $0.2 \mathrm{THz}$, which would then need even higher THz energies as a driver, since corresponding structures are larger [2]. Here, we report generation of single-cycle THz pulses with a center frequency of $0.28 \mathrm{THz}$, an energy of $0.2 \mathrm{~mJ}$ and a conversion efficiency of $0.5 \%$ using 40-mJ, 1020-nm, 1-ps pulses from a cryogenic Yb:YLF laser generated with the TPFT method in cryogenically cooled Lithium Niobate. Numerical simulations show, that further scaling to $1 \%$ efficiency with 100 $\mathrm{mJ}$ driver pulses is possible and should result in $1 \mathrm{~mJ} \mathrm{THz}$ pulses at about $0.3 \mathrm{THz}$ wavelength. Cryogenic cooling of Lithium Niobate lowers the absorption at $\mathrm{THz}$ frequencies. The conversion efficiency is limited due to break-up of the driver pulses after excessive cascading [3].

For multi-cycle or narrowband $\mathrm{THz}$ generation, difference frequency generation (DFG) in cryogenically cooled periodically poled lithium niobate (PPLN) crystals using quasi-phase matching promises high conversion efficiency of several percent. Cryogenic cooling allows the use of long crystals and cascading does not lead to break-up of the driver pulses as in the TPFT case due to collinear interaction of the waves [4]. DFG using highenergy broadband Ti:Sappire pulses and the chirp and delay method to match the difference frequency to the quasiphase matching period resulted in $40 \mu \mathrm{J}$ pulses at $0.5 \mathrm{THz}$ and $260 \mathrm{ps}$ duration [5]. The use of large aperture crystals fabricated by the Taira group and linearization of the difference frequency between the chirped and delayed pulses lead to further improvements by more than an order of magnitude to produce $460 \mu \mathrm{J}$ of $\mathrm{THz}$ radiation with about $460 \mathrm{~mJ}$ of laser pulses [6]. Cascaded optical parametric generation is proposed enabling multi-\% level conversion efficiency eventually producing up to $50 \mathrm{~mJ}$ of multi-cylce $\mathrm{THz}$ pulses from a 1-J laser pulse to drive a $\mathrm{THz}$ accelerator using dielectrically loaded metal waveguides is discussed [7].

\section{THz guns and accelerators}

Over the last years, several key demonstrations have shown the feasibility of a THz accelerator technology. A proof of principle $\mathrm{THz}$ acceleration experiment based on laser-generated single-cycle $\mathrm{THz}$ pulses with $10 \mu \mathrm{J}$ pulse energy 
at $0.45 \mathrm{THz}$ has been performed recently [8]. First $\mathrm{THz}$ guns based on a parallel plate $\mathrm{THz}$ waveguide have been reported producing quasi-monochromatic electron bunches with a few percent energy spread around $400 \mathrm{eV}$ mean energy [9]. More advanced THz gun structures driven by either single-cycle or multi-cycle $\mathrm{THz}$ pulses have been
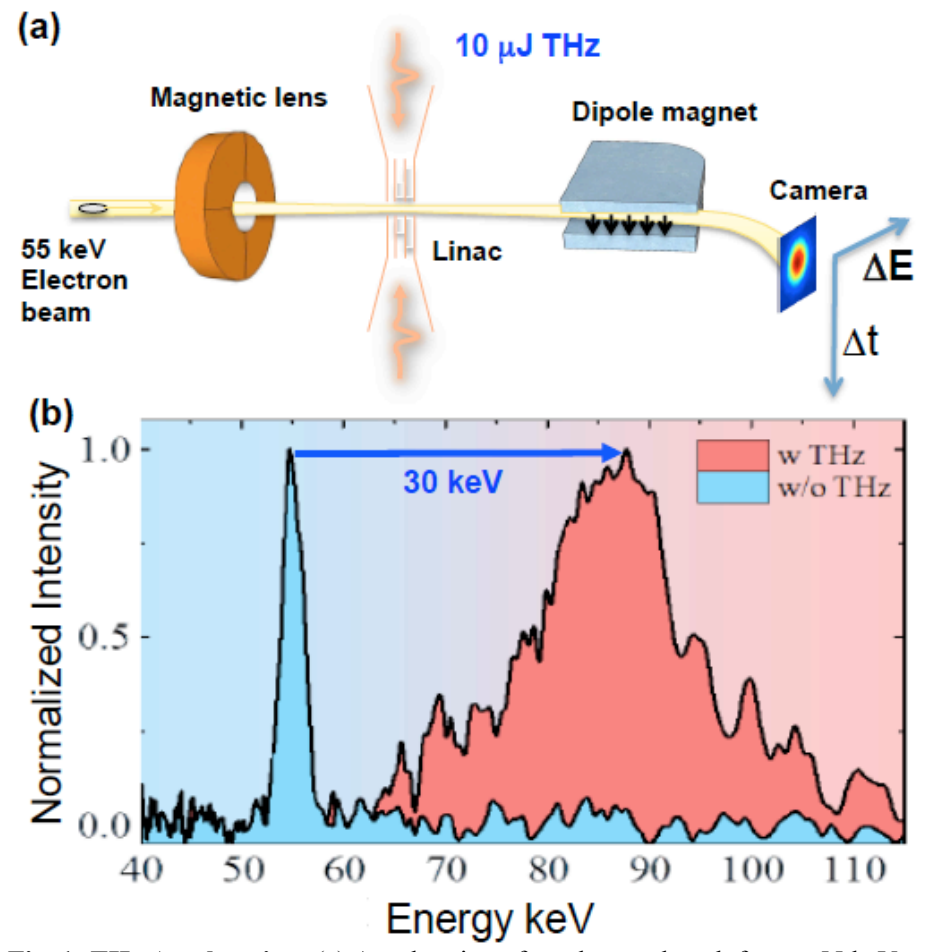

Fig. 1: THz Acceleration: (a) Acceleration of an electron bunch from a $55 \mathrm{keV}$ DC-gun using a segmented $\mathrm{THz}$ waveguide acceleration device with dipole magnet for energy measurement on MCP; (b) electron energy spectrum without (black) and with THz pulse(red). [12] proposed [10,11]. The single-cycle gun structure is a segmented $\mathrm{THz}$ waveguide device composed of an array of parallel plate waveguides with dielectric fillings to delay the $\mathrm{THz}$ pulses in each waveguide, such that when excited with a single high energy $\mathrm{THz}$ pulse jointly, a tilted pulse front is created at the interaction point with electrons. This leads to continuous acceleration of the electron bunch in the interaction areas, see Fig. 1 (a). To demonstrate this single cycle accelerator concept, we used a UV-photo-triggered, 55 $\mathrm{kV}$ DC-gun to inject an electron bunch into the accelerating structure. The accelerating structure is driven by two single-cycle $\mathrm{THz}$ pulses with about $10 \mu \mathrm{J}$ of $\mathrm{THz}$ energy. Fig. 1 (b) shows the energy spectrum of the accelerated electron bunch, measured by a dipole magnet and an MCP. The spectrum measured with and without the $\mathrm{THz}$ pulses are shown. The $\mathrm{THz}$ accelerator boosts the average electron energy by $35 \mathrm{keV}$ and significantly broadens the energy distribution due to the long electron bunch from the DC-gun of about $500 \mathrm{fs}$. The long pulse fills significant portion of the about 3 ps period accelerating $\mathrm{THz}$ field cylce. For acceleration, the two $\mathrm{THz}$ pulses are injected such that the electric fields constructively interfere in the center of the structure, and, in forward direction the transveres magnetic fields are canceled.

\section{THz streaker}

If the THz pulses in Fig. 1(a) are delayed with respect to each other by half a THz wavelength, the electric fields cancel in the center of the device and the transverse magnetic fields add, which leads to a transverse deflection of the electron bunch. This operation mode can be used for streaking of the electron bunch to measure its duration [12]. When electrons sweep the positive cycle of the B-field, the deflection is maximized and the beam profile is also best preserved. In this mode, electron beams can be precisely steered by varying the THz pulse energy. Here, we achieved continuous control of the beam angle over a range of $70 \mathrm{mrad}$ which was limited by the aperture of the device (Fig. 2).

Electrons sweeping the zero crossing cycle of the $\mathrm{THz} \mathrm{B}$-field, on the other hand, experience a strong deflection as a function of delay time enabling the measurement of the temporal bunch profiles of very short bunches by mapping (or "streaking") them onto the spatial dimension of a detector. To test this concept, a first STEAM device was used in compression mode (as described above) to provide electrons of varying bunch durations at a second, downstream STEAM device which analyzed the temporal profiles by streaking. Figure 2(a) shows raw images of a temporally-long electron beam with the $\mathrm{THz}$ streaking field switched on and off. Streaking "deflectograms," generated by plotting a lineout of the spatial charge distribution along the streaking dimension as a function of delay relative to the $\mathrm{THz}$ field, are shown in Fig. 2(b) for compressed and uncompressed electron bunches. The degree of streaking, indicated by the vertical extent of the deflectogram, depends clearly on the bunch duration and on the phase of the THz field, as expected. For a THz energy of $\sim 2 \times 6 \mathrm{~mJ}$ coupled into the device, a maximum deflection rate of $>140 \mu \mathrm{rad} / \mathrm{fs}$ was achieved, corresponding to a temporal resolution below $10 \mathrm{fs}$. The 
resolution was limited here by the $350 \mu \mathrm{m}$ size of the unstreaked beam. These results represent a new record in THzbased streaking gradient as well as the first use of $\mathrm{THz} \mathrm{B}$-fields for deflection and streaking.

a)

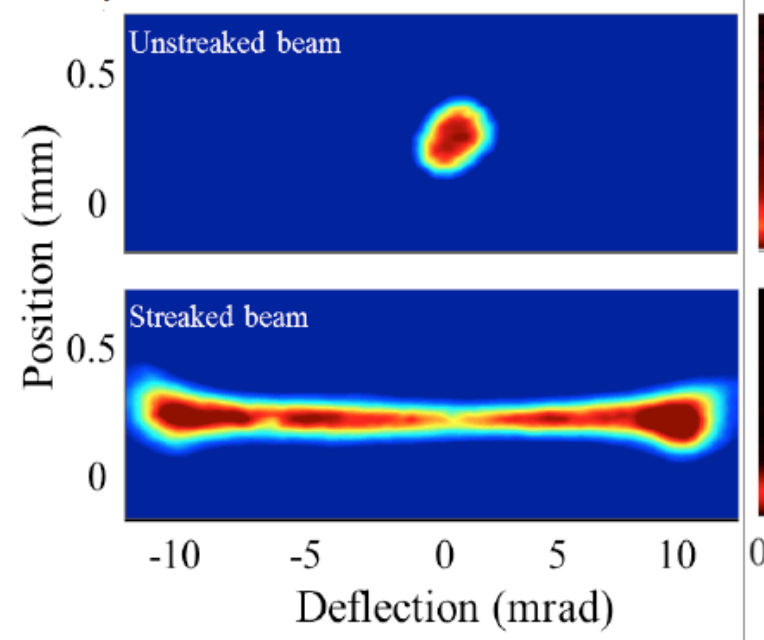

b)

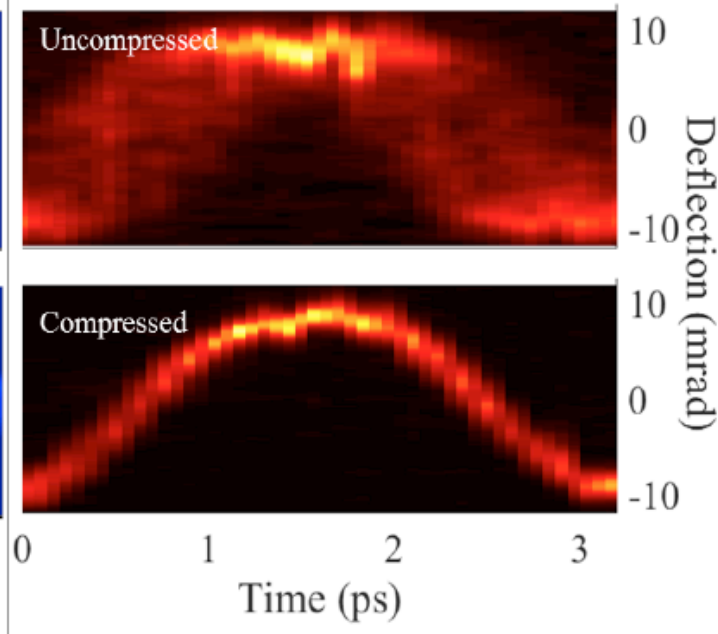

Fig. 2: Terahertz streak camera. (A) Measured images of the electron beam on the MCP-detector with and without the terahertz deflection field. (B) Time-dependent deflection diagram measured by varying the delay between the arrival time of electron bunch and the deflecting terahertz pulse for initially compressed and uncompressed electron bunches. This demonstration was performed with a bunch charge of $\sim 1$

$\mathrm{fC}$, except for the maximum streaked beam (a), where a bunch charge of $\sim 10 \mathrm{fC}$ is used in order to get a long pulse for demonstration.

$\mathrm{THz}$ based electron pulse compression and streaking has been shown earlier by Kealhofer et al. [13] using the electric field components, ultralow charge bunches with nanofabricated butterfly shaped devices.

\section{Conclusions}

Currrent work is geared towards pushing laser based $\mathrm{THz}$ generation to the multi-mJ level using Joule level, halfnanosecond laser pulses with optimized pulse formats [4]. Such high energy $\mathrm{THz}$ pulses approaching $20 \mathrm{~mJ}$ in a dielectically loaded metal waveguide can accelerate electrons to the 10-20 MeV level, energetic enough to enable in a first step hard X-ray generation via inverse Compton scattering [13].

\section{References}

[1] J. Hebling, G. Almasi, I. Z. Kozma, J. Kuhl, Opt. Exp. 10, 1161 (2012).

[2] J. A. Fülop, Z. Ollmann, C. Lombosi, C. Skrobol, S. P. Klingebiel, L. Palfalvi, F. Krausz, S. Karsch, J. Hebling, Opt. Exp. 22, 20155 (2014).

[3] K. Ravi, W. R. Huang, S. Carbajo, X. Wu and F. X. Kärtner, Opt. Exp. 22, 20239 (2014).

[4] K. Ravi, et al. Opt. Lett. 41, 3806 (2016) and Opt. Lett. 42, pp. 25582 (2016).

[5] F. Ahr, S. W. Jolly, N. H. Matlis, S. Carbajo. T. Kroh, K. Ravi, D. N. Schimpf, J. Schulte, H. Ishizuki, T. Taira, A. Maier and F. X. Kärtner, Opt. Lett. 42, 2118 (2017).

[6] S. W. Jolly, F. Ahr, N. H. Matlis, V, Leroux, T, Eichner, K. Ravi, H. Ishizuki, T. Taira, F. X. Kärtner abd A. R. Maier, this conference.

[7] L. J. Wong, A. Fallahi and F. X. Kärtner, Opt. Express 21, 9792 (2013)

[8] E. A. Nanni, W. R. Huang, K. Ravi, A. Fallahi, G. Moriena, R. J. Miller, and F. X. Kärtner, Nat. Communications 6, p. 8486 (2015).

[9] W. R. Huang, et al, Optica 3, p. 1209 (2016).

[10] A. Fallahi, M. Fakhari, A. Yahaghi, M. Arrieta, and F. X. Kärtner, Phys. Rev. Accelerators and Beams 19, 081302 (2016).

[11] M. Fakahri, A. Fallahi and F. X. Kärtner, to appear in Phys. Rev. Accelerators and Beams 19, 081302 (2017).

[12] D. Zhang, A. Fallahi, M. Hemmer, X. Wu, M. Fakhari, Y. Hua, H. Cankaya, A.-L. Calendron, L. Zapata, N. H. Matlis, and F. X. Kärtner, arXiv 1711.03024. (2017)

[13] C. Kealhofer, W. Schneider, D. Ehberger, A. Ryabov, F. Krausz, P. Baum, Science 359, 459 (2016).

[14] F. X. Kärtner et al. doi:10.1016/j.nima.2016.02.080. 